\title{
Multi-category parallel models in the design of surveys with sensitive questions
}

\author{
Yin LiU AND GuO-Liang Tian*
}

In the past few years, several non-randomized response (NRR) designs were introduced in sample surveys with sensitive questions. However, existing NRR models (e.g., the crosswise model, the triangular model, the hidden sensitive model and the multi-category triangular model) have certain limitations in applications, for example, they can only be applied to a situation where at least one of the population categories of interest is non-sensitive. In this paper, we propose a new NRR multi-category parallel model with a better degree of privacy protection and a wider application range, where all population categories of interest can be sensitive or one of them can be totally non-sensitive. Likelihoodbased inferences for parameters of interest are developed. In addition, an important special case of the multi-category parallel model is studied to test the association of two sensitive binary variables. Furthermore, theoretic comparisons show that the multi-category parallel model is more efficient than the multi-category triangular model for some cases. An example on the study of association between the number of sex partners and annual income is used to illustrate the proposed method.

KEYWORDS AND PHRASES: Chi-squared test, Likelihood ratio test, Multi-category parallel model, Multi-category triangular model, Non-randomized response technique.

\section{INTRODUCTION}

In the past few decades, the sample survey technique has played an important role in epidemiological, psychological, medical and social studies and is indispensable in assisting researchers to make statistical inferences and in guiding them to establish a meaningful decision. However, in surveys involving sensitive information or highly private questions (e.g., sexual behavior, drug-taking, tax evasion, cheating on exams, gambling and so on), problems often arise when sensitive questions are asked directly. For example, some respondents may refuse to answer or provide false answers in order to protect their privacy. Statistical inferences based on these inaccurate survey data are in general unreliable.

For a single sensitive question with binary answers ('yes' or 'no'), Warner [15] proposed a randomized response (RR)

\footnotetext{
${ }^{*}$ Corresponding author.
}

method for partially overcoming aforementioned problems while protecting respondents' privacy. Such a technique encourages interviewees to provide truthful responses, avoiding a non-response or false answer. Abul-Ela et al. [1] extended the Warner model from the dichotomous case to the multichotomous case. Another extension of the Warner model was made by Bourke and Dalenius [3], in which a Latin square design was suggested. In addition, Eriksson [4] proposed an unrelated question RR model, which could be used to estimate the proportions of $m(>2)$ mutually exclusive sensitive groups (up to $m-1$ sensitive subclasses) only using one sample. Bourke [5] considered another unrelated question model to estimate the proportions of $m$ mutually exclusive groups with $k(1 \leq k \leq m-1)$ groups containing sensitive information. If the distribution of the unrelated characteristic is known, only one sample is needed. Because of the use of one or two randomized devices (RDs), all RR models have some limitations including the lack of reproducibility, of trust, and of cost control.

Recently, without using any RDs some investigators proposed several non-randomized response (NRR) models $[13,14,17,11]$, which could overcome some of the limitations with RR designs. Despite greater advances, all NRR models including the multi-category triangular model [11] require that at least one of the population categories of interest be non-sensitive. For example, in some surveys, we may be interested in estimating the proportions of population groups associated with sensitive questions such as the number of sexual partners $(\leq 3,4-6,>7)$, or days of illegal drug usage in the last month $(\leq 1,2$, or $\geq 3)$, and so on. First, as the unique NRR model dealing with the case of $m(m \geq 3)$ groups, the existing multi-category triangular model cannot be applied to such situations where each subclass (denoted by $\{Y=i\}$ for $i=1, \ldots, m$ ) is sensitive. Second, the multi-category triangular model still has a lower efficiency for some cases. Third, the newly developed parallel model of Tian [12] can only deal with the case of $m=2$ groups where both $\{Y=0\}$ and $\{Y=1\}$ could be sensitive. Therefore, these limitations with NRR models motivate us to further develop a new non-randomized multicategory parallel model, which is an extension of the NRR parallel model.

This article is organized as follows. In Section 2, we propose the survey design for the multi-category parallel model with a wider application range. Section 3 presents the max- 
Table 1. The multi-category parallel model and the corresponding cell probabilities

\begin{tabular}{|c|c|c|c|c|c|c|}
\hline$\overline{\overline{\text { Category }}}$ & $\bar{W} W=0$ & $W=1$ & Category & $\bar{W}=0$ & $\bar{W}=1$ & Marginal \\
\hline$U=1$ & 0 & & $U=1$ & $p_{1}(1-q)$ & & $p_{1}$ \\
\hline$U=2$ & $\triangle$ & & $U=2$ & $p_{2}(1-q)$ & & $p_{2}$ \\
\hline$\vdots$ & • & & $\vdots$ & & & : \\
\hline$U=m$ & - & & $U=m$ & $p_{m}(1-q)$ & & $p_{m}$ \\
\hline$Y=1$ & & 0 & $Y=1$ & & $\pi_{1} q$ & $\pi_{1}$ \\
\hline$Y=2$ & & $\triangle$ & $Y=2$ & & $\pi_{2} q$ & $\pi_{2}$ \\
\hline$\vdots$ & & & $\vdots$ & & $\vdots$ & $\vdots$ \\
\hline \multirow[t]{2}{*}{$Y=m$} & & - & $Y=m$ & & $\pi_{m} q$ & $\pi_{m}$ \\
\hline & & & Marginal & $1-q$ & $q$ & 1 \\
\hline
\end{tabular}

Note: Please truthfully link the two circles by a straightline if you belong to $\{U=1, W=0\} \cup\{Y=1, W=1\}$, or link the two triangles by a straightline if you belong to $\{U=2, W=0\} \cup\{Y=2, W=1\}, \ldots$, or link the two dots by a straightline if you belong to $\{U=m, W=0\} \cup\{Y=m, W=1\}$.

imum likelihood estimates (MLEs) and two bootstrap confidence intervals (CIs) of the parameters of interest for small to moderate sample sizes. In addition, two asymptotic CIs of parameters are also constructed for large sample sizes. An important special case $(m=4)$ for the multi-category parallel design is studied in Section 4. In Section 5, we compare the efficiency between the multi-category parallel model and the multi-category triangular model. In Section 6 , an example on the study of association between the number of sex partners and annual income is used to illustrate the proposed method. Finally, we conclude with a discussion in Section 7 .

\section{THE SURVEY DESIGN FOR THE MULTI-CATEGORY PARALLEL MODEL}

Consider a sensitive question $Q_{Y}$ (e.g., how many sex partners do you have within a certain period?) with $m$ possible answers (e.g., $0-3,4-6$ or $\geq 7$ ), which classify the target population into $m$ mutually exclusive categories and each category has a certain degree of a sensitive attribute. Let $Y$ denote the categorical random variable associated with the question $Q_{Y}$ and $\{Y=i\}$ denote that a person in the target population belongs to the $i$-th category $(i=1, \ldots, m)$. The purpose here is to estimate the proportions $\pi_{i}=\operatorname{Pr}\{Y=i\}$ for $i=1, \ldots, m$. Let

$$
\mathbb{T}_{m} \hat{=}\left\{\left(x_{1}, \ldots, x_{m}\right)^{\top}: x_{i} \geq 0, i=1, \ldots, m, \sum_{i=1}^{m} x_{i}=1\right\}
$$

obviously, we have $\boldsymbol{\pi}=\left(\pi_{1}, \ldots, \pi_{m}\right)^{\top} \in \mathbb{T}_{m}$.

To carry out the survey for which each category includes a sensitive attribute, we require an introduction of the nonsensitive dichotomous variate $W$ and another non-sensitive multichotomous variate $U$ so that the three variables $W, U$ and $Y$ are mutually independent with known proportions $q=\operatorname{Pr}\{W=1\}$ and

$$
p_{i}=\operatorname{Pr}\{U=i\}, \quad i=1, \ldots, m .
$$

For example, when $m=4$, we may define $W=0$ if the respondent's birthday is in the first half of a month and $W=1$ otherwise. Similarly, we define $U=i$ if the respondent was born in the $i$-th quarter of a year $(i=1, \ldots, 4)$. Hence, it is reasonable to assume that $q \approx 0.5$ and $p_{i} \approx 0.25$ for each $i$. Some practical guidelines in choosing the two non-sensitive variables $W$ and $U$ are given in Section 7. The interviewer may design the questionnaire in the format as shown at the left-hand side of Table 1 and ask the interviewee to truthfully link the two circles by a straight line if he/she belongs to one of the two circles (i.e., $\{U=1, W=0\}$ or $\{Y=1, W=1\})$; or to connect the two triangles by a straight line if he/she belongs to one of the two triangles (i.e., $\{U=2, W=0\}$ or $\{Y=2, W=1\}$ ); . ; or to connect the two dots by a straightline if he/she belongs to one of the two dots (i.e., $\{U=m, W=0\}$ or $\{Y=m, W=1\}$ ). Note that all $\{W=0\},\{W=1\}$, and $\{U=i\}$ are nonsensitive subclasses, thus

$$
\{U=i, W=0\} \cup\{Y=i, W=1\}, \quad i=1, \ldots, m,
$$

are also non-sensitive subclasses. Therefore, the respondent's privacy is well protected, and the interviewer does not have information on whether the interviewee belongs to the sensitive class or not. We call this the multi-category parallel model. The right-hand side of Table 1 shows the corresponding cell probabilities. Since the three random variables $W, U$ and $Y$ are independent, the joint probability is the product of two corresponding marginal probabilities.

For those who may not completely understand the questionnaire shown in Table 1, we can formulate the survey design of the multi-category parallel model in another manner. For example, let $m=4$ and define $Y=1,2,3$ or 4 if the 
number of days of the illegal drug usage in the last month for a respondent is $0-1,2,3$ or $\geq 4$. Thus, the 4-category parallel model can be re-formulated in the following way:

$1^{\circ}$ If your birthday is in the first half of a month (i.e., $W=0$ ), please answer ' 1 ' (i.e., $U=1$ ), or ' 2 ' (i.e., $U=2$ ), or ' 3 ' (i.e., $U=3$ ), or ' 4 ' (i.e., $U=4$ ) to the question: In which quarter of the year is your birthday?

$2^{\circ}$ If your birthday is in the second half of a month (i.e., $W=1$ ), please answer ' 1 ' (i.e., $Y=1$ ), or '2' (i.e., $Y=2$ ), or '3' (i.e., $Y=3$ ), or '4' (i.e., $Y=4$ ) to the question: How many days in the last month have you used illegal drugs?

\section{MAXIMUM LIKELIHOOD INFERENCES}

\subsection{MLEs of parameters via the EM algorithm}

Suppose we conduct a sample survey with $n$ questionnaires and observe $n_{1}$ respondents connecting the two circles, $n_{2}$ respondents connecting the two triangles, ..., and $n_{m}$ respondents connecting the two dots (see, Table 1). Let $Y_{\text {obs }}=\left\{n ; n_{1}, \ldots, n_{m}\right\}$ denote the observed data, where $n=\sum_{i=1}^{m} n_{i}$. Hence, the observed-data likelihood function for $\boldsymbol{\pi}=\left(\pi_{1}, \ldots, \pi_{m}\right)^{\top}$ is

$$
L_{\mathrm{MP}}\left(\boldsymbol{\pi} \mid Y_{\mathrm{obs}}\right)=\left(\begin{array}{c}
n \\
n_{1}, \ldots, n_{m}
\end{array}\right) \prod_{i=1}^{m}\left[p_{i}(1-q)+\pi_{i} q\right]^{n_{i}}
$$

where the subscript 'MP' refers to the 'multi-category parallel' model. We employ the EM algorithm [7] to calculate the MLEs of $\left\{\pi_{i}\right\}_{i=1}^{m}$ by introducing the latent vector $\boldsymbol{z}=\left(z_{1}, \ldots, z_{m}\right)^{\top}$, where $z_{i}$ denotes the number of respondents belonging to the sensitive subclass $\{Y=i, W=1\}$. We denote the complete data by $Y_{\text {com }}=\left\{Y_{\text {obs }}, \boldsymbol{z}\right\}$. Note that $\left\{p_{i}\right\}_{i=1}^{m}$ and $q$ are known, the complete-data likelihood function for $\pi$ is

$$
L_{\mathrm{MP}}\left(\boldsymbol{\pi} \mid Y_{\mathrm{obs}}, \boldsymbol{z}\right) \propto \prod_{i=1}^{m}\left[p_{i}(1-q)\right]^{n_{i}-z_{i}}\left(\pi_{i} q\right)^{z_{i}} \propto \prod_{i=1}^{m} \pi_{i}^{z_{i}} .
$$

Therefore, the M-step is to calculate the complete-data MLEs of $\left\{\pi_{i}\right\}_{i=1}^{m}$, which are given by

$$
\hat{\pi}_{i}=\frac{z_{i}}{z_{1}+\cdots+z_{m}}, \quad i=1, \ldots, m .
$$

Since the conditional predictive density is

$$
\begin{aligned}
f\left(\boldsymbol{z} \mid Y_{\mathrm{obs}}, \boldsymbol{\pi}\right) \\
\quad=\prod_{i=1}^{m} f\left(z_{i} \mid Y_{\mathrm{obs}}, \pi_{i}\right) \\
\quad=\prod_{i=1}^{m} \operatorname{Binomial}\left(z_{i} \mid n_{i}, \frac{\pi_{i} q}{p_{i}(1-q)+\pi_{i} q}\right),
\end{aligned}
$$

the E-step is to replace $z_{i}$ in (3.3) by its conditional expectation

$$
E\left(z_{i} \mid Y_{\mathrm{obs}}, \pi_{i}\right)=\frac{n_{i} \pi_{i} q}{p_{i}(1-q)+\pi_{i} q}, \quad i=1, \ldots, m .
$$

\subsection{Two bootstrap confidence intervals of parameters}

We utilize the bootstrap method to derive the corresponding CIs of $\left\{\pi_{i}\right\}_{i=1}^{m}$. Based on the obtained MLE $\hat{\boldsymbol{\pi}}_{\mathrm{MP}}=$ $\left(\hat{\pi}_{\mathrm{MP} 1}, \ldots, \hat{\pi}_{\mathrm{MP} m}\right)^{\top}$ of $\boldsymbol{\pi}$, we could generate

$\left(n_{1}^{*}, \ldots, n_{m}^{*}\right)^{\top} \sim$

$\operatorname{Multinomial}\left(n ; p_{1}(1-q)+q \hat{\pi}_{\mathrm{MP} 1}, \ldots, p_{m}(1-q)+q \hat{\pi}_{\mathrm{MP} m}\right)$.

For the bootstrap sample $\left\{n_{1}^{*}, \ldots, n_{m}^{*}\right\}$, we can compute the bootstrap replication $\hat{\pi}_{\mathrm{MP} i}^{*}$ via the EM algorithm (3.3) and (3.5) by replacing $\left\{n_{1}, \ldots, n_{m}\right\}$ with $\left\{n_{1}^{*}, \ldots, n_{m}^{*}\right\}$. Independently repeating this process $G$ times, we obtain $G$ bootstrap replications $\left\{\hat{\pi}_{\mathrm{MP} i}^{*}(g)\right\}_{g=1}^{G}$. Therefore, the standard error, $\operatorname{Se}\left(\hat{\pi}_{\mathrm{MP} i}\right)$, of $\hat{\pi}_{\mathrm{MP} i}$ can be estimated by the sample standard deviation of the $G$ replications, i.e.

$$
\begin{aligned}
& \widehat{\operatorname{Se}}\left(\hat{\pi}_{\mathrm{MP} i}\right) \\
& \quad=\left\{\frac{1}{G-1} \sum_{g=1}^{G}\left[\hat{\pi}_{\mathrm{MP} i}^{*}(g)-\frac{\hat{\pi}_{\mathrm{MP} i}^{*}(1)+\cdots+\hat{\pi}_{\mathrm{MP} i}^{*}(G)}{G}\right]^{2}\right\}^{\frac{1}{2}} .
\end{aligned}
$$

If $\left\{\hat{\pi}_{\mathrm{MP} i}^{*}(g)\right\}_{g=1}^{G}$ is approximately normally distributed, a $(1-\alpha) 100 \%$ bootstrap CI for $\pi_{i}$ is given by

$$
\left[\hat{\pi}_{\mathrm{MP} i}-z_{\alpha / 2} \widehat{\operatorname{Se}}\left(\hat{\pi}_{\mathrm{MP} i}\right), \hat{\pi}_{\mathrm{MP} i}+z_{\alpha / 2} \widehat{\operatorname{Se}}\left(\hat{\pi}_{\mathrm{MP} i}\right)\right],
$$

where $z_{\alpha}$ is the upper $\alpha$-th quantile of the standard normal distribution. Alternatively, if $\left\{\hat{\pi}_{\mathrm{MP} i}^{*}(g)\right\}_{g=1}^{G}$ is non-normally distributed or the bootstrap CI (3.7) is beyond the unit interval $(0,1)$, a $(1-\alpha) 100 \%$ bootstrap CI of $\pi_{i}$ can be obtained by

$$
\left[\hat{\pi}_{\mathrm{MP} i, \mathrm{BL}}, \hat{\pi}_{\mathrm{MP} i, \mathrm{BU}}\right],
$$

where $\hat{\pi}_{\mathrm{MP} i, \mathrm{BL}}$ and $\hat{\pi}_{\mathrm{MP} i, \mathrm{BU}}$ are the $100(\alpha / 2)$ and $100(1-\alpha / 2)$ percentiles of $\left\{\hat{\pi}_{\mathrm{MP} i}^{*}(g)\right\}_{g=1}^{G}$, respectively.

\subsection{Explicit solutions to the valid estimators}

Although the resulting MLE $\hat{\pi}_{\mathrm{MP}}$ via the EM algorithm (3.3) and (3.5) definitely belongs to $\mathbb{T}_{m}$, we can only obtain a numerical solution to $\hat{\boldsymbol{\pi}}_{\mathrm{MP}}$. In addition, the variancecovariance matrix of $\hat{\boldsymbol{\pi}}_{\mathrm{MP}}$ does not have a closed-form expression. However, for some cases, we can obtain explicit solutions to $\hat{\boldsymbol{\pi}}_{\mathrm{MP}}$ and its variance-covariance matrix.

From (3.1), the log-likelihood function is given by

$$
\ell_{\mathrm{MP}}\left(\boldsymbol{\pi} \mid Y_{\mathrm{obs}}\right)=c+\sum_{i=1}^{m} n_{i} \log \left[p_{i}(1-q)+\pi_{i} q\right],
$$


where $c$ is a constant not depending on $\boldsymbol{\pi}$. Let $\partial \ell_{\mathrm{MP}}\left(\boldsymbol{\pi} \mid Y_{\mathrm{obs}}\right) / \partial \pi_{i}=0$, an alternative estimator of $\boldsymbol{\pi}$ is given by

$$
\begin{aligned}
\hat{\boldsymbol{\pi}}_{v} & =\left(\hat{\pi}_{v 1}, \ldots, \hat{\pi}_{v m}\right)^{\top} \\
& =\left(\frac{n_{1} / n-p_{1}(1-q)}{q}, \ldots, \frac{n_{m} / n-p_{m}(1-q)}{q}\right)^{\top}
\end{aligned}
$$

Although $\hat{\pi}_{v i}$ is an unbiased estimator of the true proportion $\pi_{i}, \hat{\boldsymbol{\pi}}_{v}$ may not belong to $\mathbb{T}_{m}$. For example, let $m=4, p_{1}=$ $\cdots=p_{4}=0.25, q=1 / 3$ and $\left(n_{1}, \ldots, n_{4}\right)^{\top}=(15,19,7,9)^{\top}$, then

$$
\hat{\boldsymbol{\pi}}_{v}=(0.40,0.64,-0.08,0.04)^{\top} \notin \mathbb{T}_{4}
$$

In this paper, the estimator $\hat{\boldsymbol{\pi}}_{v}$ given by (3.9) is said to be valid if $\hat{\boldsymbol{\pi}}_{v} \in \mathbb{T}_{m}$. Clearly, if $\hat{\boldsymbol{\pi}}_{v}$ specified by (3.9) is a valid estimator of $\boldsymbol{\pi}$, then $\hat{\boldsymbol{\pi}}_{v}=\hat{\boldsymbol{\pi}}_{\mathrm{MP}}$. In the following discussion, we only consider the case of valid estimators.

Note that $\left(n_{1}, \ldots, n_{m}\right)^{\top} \sim \operatorname{Multinomial}_{m}(n, \boldsymbol{\lambda})$, where $\boldsymbol{\lambda}=\left(\lambda_{1}, \ldots, \lambda_{m}\right)^{\top} \in \mathbb{T}_{m}$

$$
\lambda_{i}=p_{i}(1-q)+\pi_{i} q, \quad i=1, \ldots, m
$$

Let $\hat{\boldsymbol{\lambda}}=\left(\hat{\lambda}_{1}, \ldots, \hat{\lambda}_{m}\right)^{\top}=\left(n_{1} / n, \ldots, n_{m} / n\right)^{\top}$ denote the MLE of $\boldsymbol{\lambda}$. Then (3.9) can be rewritten in the vector form as

$$
\hat{\boldsymbol{\pi}}_{\mathrm{MP}}=\left(\hat{\pi}_{\mathrm{MP} 1}, \ldots, \hat{\pi}_{\mathrm{MP} m}\right)^{\top}=\frac{\hat{\boldsymbol{\lambda}}-(1-q) \boldsymbol{p}}{q},
$$

where $\boldsymbol{p}=\left(p_{1}, \ldots, p_{m}\right)^{\top}$. Thus, the variance-covariance matrix of $\hat{\pi}_{\mathrm{MP}}$ is given by

$$
\begin{aligned}
\operatorname{Var}\left(\hat{\boldsymbol{\pi}}_{\mathrm{MP}}\right) & =\frac{1}{q^{2}} \operatorname{Var}(\hat{\boldsymbol{\lambda}}) \\
& =\frac{1}{n q^{2}}\left(\begin{array}{cccc}
\lambda_{1}\left(1-\lambda_{1}\right) & -\lambda_{1} \lambda_{2} & \cdots & -\lambda_{1} \lambda_{m} \\
-\lambda_{2} \lambda_{1} & \lambda_{2}\left(1-\lambda_{2}\right) & \cdots & -\lambda_{2} \lambda_{m} \\
\vdots & \vdots & \ddots & \vdots \\
-\lambda_{m} \lambda_{1} & -\lambda_{m} \lambda_{2} & \cdots & \lambda_{m}\left(1-\lambda_{m}\right)
\end{array}\right) .
\end{aligned}
$$

\subsection{Three asymptotic confidence intervals of parameters for large sample sizes}

From (3.12), it is not difficult to show the following result.

Theorem 1. Let

$$
\widehat{\operatorname{Var}}\left(\hat{\pi}_{\mathrm{MP} i}\right)=\frac{\hat{\lambda}_{i}\left(1-\hat{\lambda}_{i}\right)}{(n-1) q^{2}}, \quad i=1, \ldots, m .
$$

Then, we have

$$
\widehat{\operatorname{Var}}\left(\hat{\pi}_{\mathrm{MP} i}\right)=\frac{\hat{\pi}_{\mathrm{MP} i}\left(1-\hat{\pi}_{\mathrm{MP} i}\right)}{n-1}+\frac{(1-q) f\left(\hat{\pi}_{\mathrm{MP} i}, p_{i}, q\right)}{(n-1) q^{2}},
$$

140 Y. Liu and G. Tian where $f\left(\pi_{i}, p_{i}, q\right) \hat{=} q\left(1-2 p_{i}\right) \pi_{i}+p_{i}\left(1-p_{i}+q p_{i}\right)$, and it $i s$ an unbiased estimator of $\operatorname{Var}\left(\hat{\pi}_{\mathrm{MP} i}\right)=\lambda_{i}\left(1-\lambda_{i}\right) /\left(n q^{2}\right)$, $i=1, \ldots, m$.

Based on the property of MLE for large sample sizes, we have

$$
\frac{\hat{\pi}_{\mathrm{MP} i}-\pi_{i}}{\sqrt{\widehat{\operatorname{Var}}\left(\hat{\pi}_{\mathrm{MP} i}\right)}} \dot{\sim} N(0,1), \quad \text { as } n \rightarrow \infty, i=1, \ldots, m .
$$

Thus, an asymptotic $(1-\alpha) 100 \%$ Wald CI for $\pi_{i}$ is given by

$$
\left[\hat{\pi}_{\mathrm{MP} i}-z_{\alpha / 2} \sqrt{\widehat{\operatorname{Var}}\left(\hat{\pi}_{\mathrm{MP} i}\right)}, \quad \hat{\pi}_{\mathrm{MP} i}+z_{\alpha / 2} \sqrt{\widehat{\widehat{\operatorname{Var}}\left(\hat{\pi}_{\mathrm{MP} i}\right)}}\right] .
$$

If the lower bound of the Wald CI in (3.14) is less than zero or the upper bound is larger than one, then the Wald CI is useless. For such cases, according to the Central Limit Theorem, we can establish an asymptotic $(1-\alpha) 100 \%$ Wilson (score) CI of $\pi_{i}$ based on

$$
\begin{aligned}
& 1-\alpha= \operatorname{Pr}\left\{\left|\frac{\hat{\pi}_{\mathrm{MP} i}-\pi_{i}}{\sqrt{\operatorname{Var}\left(\hat{\pi}_{\mathrm{MP} i}\right)}}\right| \leq z_{\alpha / 2}\right\} \\
&= \operatorname{Pr}\left\{\left(\hat{\pi}_{\mathrm{MP} i}-\pi_{i}\right)^{2} \leq z_{\alpha / 2}^{2} \operatorname{Var}\left(\hat{\pi}_{\mathrm{MP} i}\right)\right\} \\
& \stackrel{(3.12)}{=} \operatorname{Pr}\left\{\left(\hat{\pi}_{\mathrm{MP} i}-\pi_{i}\right)^{2} \leq \frac{z_{\alpha / 2}^{2}}{n}\left[\pi_{i}\left(1-\pi_{i}\right)\right.\right. \\
&\left.\left.+\frac{\left.\left.(1-q) f\left(\pi_{i}, p_{i}, q\right)\right]\right\}}{q^{2}}\right]\right\} \\
&=\operatorname{Pr}\left\{\hat{\pi}_{\mathrm{MP} i}^{2}-2 \hat{\pi}_{\mathrm{MP} i} \pi_{i}+\pi_{i}^{2}\right. \\
&\left.\leq \frac{z_{\alpha / 2}^{2}\left(-\pi_{i}^{2}+\rho_{1} \pi_{i}+\rho_{2}\right)}{n}\right\} \\
&=\operatorname{Pr}\left\{\left(1+z_{*}\right) \pi_{i}^{2}-\left(2 \hat{\pi}_{\mathrm{MP} i}+z_{*} \rho_{1}\right) \pi_{i}\right. \\
&\left.\quad+\hat{\pi}_{\mathrm{MP} i}^{2}-z_{*} \rho_{2} \leq 0\right\},
\end{aligned}
$$

where $z_{*} \hat{=} z_{\alpha / 2}^{2} / n, \rho_{1} \hat{=}\left[1-2 p_{i}(1-q)\right] / q$ and $\rho_{2} \hat{=} p_{i}(1-$ $q)\left(1-p_{i}+q p_{i}\right) / q^{2}$. Solving the quadratic inequality inside the probability in (3.15), we obtain the Wilson CI given by (3.16)

$\frac{2 \hat{\pi}_{\mathrm{MP} i}+z_{*} \rho_{1} \pm \sqrt{\left(2 \hat{\pi}_{\mathrm{MP} i}+z_{*} \rho_{1}\right)^{2}-4\left(1+z_{*}\right)\left(\hat{\pi}_{\mathrm{MP} i}^{2}-z_{*} \rho_{2}\right)}}{2\left(1+z_{*}\right)}$,

which is, in general, within $[0,1]$. The Wilson CI has been shown to have better performance than the Wald CI and the exact (Clopper-Pearson) CI, see [6, 2, 10, 5] for more detail.

For sensitive responses where some of the true values $\left\{\pi_{i}\right\}$ are often small, likelihood ratio confidence intervals (LRCIs) could provide better performance than other alternatives. To construct the LRCI of $\pi_{i}(i=1, \ldots, m)$, we consider the null hypothesis $H_{0}: \pi_{i}=\pi_{i 0}$ against the alternative hypothesis $H_{1}: H_{0}$ is not true. Let $\hat{\boldsymbol{\pi}}^{R}=\left(\hat{\pi}_{1}^{R}, \ldots, \hat{\pi}_{m}^{R}\right)^{\top}$ denote the 
Table 2. The four-category parallel model and the corresponding cell probabilities

\begin{tabular}{|c|c|c|c|c|c|}
\hline Category & $W=0$ & Category & $W=0$ & $W=1$ & Marginal \\
\hline$U=1$ & \multirow{3}{*}{$\triangle$} & $U=1$ & $p_{1}(1-q)$ & & $p_{1}$ \\
\hline$U=2$ & & $U=2$ & $p_{2}(1-q)$ & & $p_{2}$ \\
\hline $\begin{array}{l}U=3 \\
U=4\end{array}$ & & $\begin{array}{l}U=3 \\
U=4\end{array}$ & $\begin{array}{l}p_{3}(1-q) \\
p_{4}(1-q)\end{array}$ & & $\begin{array}{l}p_{3} \\
p_{4}\end{array}$ \\
\hline $\bar{X}=0, Y=0$ & \multirow{5}{*}{$\triangle$} & $X=0, Y=0$ & & $\pi_{1} q$ & $\pi_{1}$ \\
\hline$X=0, Y=1$ & & $X=0, Y=1$ & & $\pi_{2} q$ & $\pi_{2}$ \\
\hline$X=1, Y=0$ & & $X=1, Y=0$ & & $\pi_{3} q$ & $\pi_{3}$ \\
\hline \multirow[t]{2}{*}{$X=1, Y=1$} & & $X=1, Y=1$ & & $\pi_{4} q$ & $\pi_{4}$ \\
\hline & & Marginal & $1-q$ & $q$ & 1 \\
\hline
\end{tabular}

restricted MLE of $\boldsymbol{\pi}$ under $H_{0}$. It can be verified that

$$
\left\{\begin{array}{l}
\hat{\pi}_{i}^{R}=\pi_{i 0}, \\
\hat{\pi}_{j}^{R}=\frac{\left[1-p_{i}(1-q)-\pi_{i 0} q\right] n_{j} /\left(n-n_{i}\right)-p_{j}(1-q)}{q},
\end{array}\right.
$$

where $j=1, \ldots, m ; j \neq i$.

When $n \rightarrow \infty$, it is well known that

$$
\Lambda\left(\pi_{i 0}\right)=-2\left\{\ell_{\mathrm{MP}}\left(\hat{\boldsymbol{\pi}}^{R} \mid Y_{\mathrm{obs}}\right)-\ell_{\mathrm{MP}}\left(\hat{\boldsymbol{\pi}}_{v} \mid Y_{\mathrm{obs}}\right)\right\} \dot{\sim} \chi^{2}(1),
$$

where $\hat{\boldsymbol{\pi}}_{v}$ denotes the unrestricted MLE of $\boldsymbol{\pi}$ specified by (3.9). Since

$$
\begin{aligned}
\Lambda\left(\pi_{i 0}\right)= & -2\left\{n_{i} \log \left[p_{i}(1-q)+\pi_{i 0} q\right]\right. \\
& +\sum_{j=1, j \neq i}^{m} n_{j} \log \left[p_{j}(1-q)+\hat{\pi}_{j}^{R} q\right] \\
& \left.-\sum_{k=1}^{m} n_{k} \log \left[p_{k}(1-q)+\hat{\pi}_{v k} q\right]\right\},
\end{aligned}
$$

it is easy to verify that $\Lambda\left(\pi_{i 0}\right)$ is a decreasing function of $\pi_{i 0}$ when $\pi_{i 0} \in\left[0, \frac{n_{i} / n-p_{i}(1-q)}{q}\right]$ and an increasing function of $\pi_{i 0}$ when $\pi_{i 0} \in\left[\frac{n_{i} / n-p_{i}(1-q)}{q}, 1\right]$. Therefore, for a given significance level $\alpha$, the $(1-\alpha) 100 \%$ LRCI for $\pi_{i}$ is given by

$$
\left[\hat{\pi}_{\mathrm{MP} i, \mathrm{LRL}}, \hat{\pi}_{\mathrm{MP} i, \mathrm{LRU}}\right],
$$

where $\hat{\pi}_{\mathrm{MP} i, \mathrm{LRL}}$ and $\hat{\pi}_{\mathrm{MP} i, \mathrm{LRU}}$ are two roots of $\pi_{i 0}$ to the following equation

$$
\Lambda\left(\pi_{i 0}\right)=\chi^{2}(\alpha, 1)
$$

where $\chi^{2}(\alpha, 1)$ denotes the upper $\alpha$-th quantile of $\chi^{2}$ distribution with one degree of freedom.

The asymptotic CIs (3.14), (3.16) and (3.18) are appropriate for the cases of large sample sizes. When $n$ is small to moderate, we could use the bootstrap CIs (3.7) and/or (3.8).

\section{A SPECIAL CASE FOR THE MULTI-CATEGORY PARALLEL MODEL}

In this section, we consider a special case of the the multicategory parallel model with four categories, which can be utilized to investigate the association of two binary sensitive variates. Some simulation studies are conducted to assess the performances of the likelihood ratio test and the chi-squared statistic by comparing their empirical type I error rates (or the actual significance levels) and powers.

\subsection{A four-category parallel model}

Let $X$ and $Y$ be two dichotomous random variables associated with two sensitive questions. For example, $X$ represents whether or not a respondent is an illegal drug user and $Y$ denotes whether a respondent is with AIDS or not. Let $X=1$ and $Y=1$ denote the sensitive characteristics of a respondent (e.g., $X=1$ if the respondent is a drug user), and $X=0$ and $Y=0$ denote the non-sensitive characteristics of a respondent (e.g., $Y=0$ if a respondent is without AIDS). Define $\pi_{1}=\operatorname{Pr}\{X=0, Y=0\}, \pi_{2}=\operatorname{Pr}\{X=0, Y=1\}$, $\pi_{3}=\operatorname{Pr}\{X=1, Y=0\}$ and $\pi_{4}=\operatorname{Pr}\{X=1, Y=1\}$. Obviously, we have $\boldsymbol{\pi}=\left(\pi_{1}, \ldots, \pi_{4}\right)^{\top} \in \mathbb{T}_{4}$. From Table 1 , the survey design for the four-category parallel model is displayed in Table 2. Two major objectives here are to collect sensitive data and to test whether or not the association exists between the two binary variates $X$ and $Y$.

\subsection{Testing hypothesis for association}

A commonly used index for measuring the association of two binary variates is the odds ratio $\psi=\pi_{1} \pi_{4} /\left(\pi_{2} \pi_{3}\right)$. Assume that we want to test $H_{0}: \psi=1$ against $H_{1}: \psi \neq 1$. The likelihood ratio statistic defined by

$$
\begin{aligned}
\Lambda_{1}= & -2\left\{\ell_{\mathrm{MP}}\left(\hat{\boldsymbol{\pi}}_{0} \mid Y_{\mathrm{obs}}\right)\right. \\
& \left.-\ell_{\mathrm{MP}}\left(\hat{\boldsymbol{\pi}}_{\mathrm{MP}} \mid Y_{\mathrm{obs}}\right)\right\} \dot{\sim} \chi^{2}(1), \quad \text { as } n \rightarrow \infty,
\end{aligned}
$$

where $\hat{\boldsymbol{\pi}}_{0}$ denotes the restricted MLE of $\boldsymbol{\pi}$ under $H_{0}$ and $\hat{\boldsymbol{\pi}}_{\mathrm{MP}}$ denotes the MLE of $\boldsymbol{\pi}$ given by (3.11). To calculate $\hat{\boldsymbol{\pi}}_{0}$, let $\pi_{x} \hat{=} \operatorname{Pr}(X=1)=\pi_{3}+\pi_{4}$ and $\pi_{y} \hat{=} \operatorname{Pr}(Y=1)=\pi_{2}+\pi_{4}$. 
If $H_{0}$ is true, i.e., $X$ and $Y$ are mutually independent, we have

$$
\left\{\begin{array}{l}
\pi_{1}=\left(1-\pi_{x}\right)\left(1-\pi_{y}\right), \\
\pi_{2}=\left(1-\pi_{x}\right) \pi_{y}, \\
\pi_{3}=\pi_{x}\left(1-\pi_{y}\right), \\
\pi_{4}=\pi_{x} \pi_{y}
\end{array}\right. \text { and }
$$

If we could obtain the restricted MLEs $\hat{\pi}_{0 x}$ of $\pi_{x}$ and $\hat{\pi}_{0 y}$ of $\pi_{y}$, from (4.2) the restricted MLEs $\hat{\pi}_{0}=\left(\hat{\pi}_{01}, \ldots, \hat{\pi}_{04}\right)^{\top}$ can be calculated as

$$
\left\{\begin{array}{l}
\hat{\pi}_{01}=\left(1-\hat{\pi}_{0 x}\right)\left(1-\hat{\pi}_{0 y}\right), \\
\hat{\pi}_{02}=\left(1-\hat{\pi}_{0 x}\right) \hat{\pi}_{0 y}, \\
\hat{\pi}_{03}=\hat{\pi}_{0 x}\left(1-\hat{\pi}_{0 y}\right), \quad \text { and } \\
\hat{\pi}_{04}=\hat{\pi}_{0 x} \hat{\pi}_{0 y} .
\end{array}\right.
$$

Recall that the number of the respondents belonging to the subclass $\{Y=i, W=1\}$ is denoted by $z_{i}$ and the frequencies $\left\{z_{i}\right\}$ are unobservable. From (3.2), the complete-data likelihood function for $\boldsymbol{\pi}$ under $H_{0}$ becomes

$$
\begin{aligned}
L_{\mathrm{MP}} & \left(\pi_{x}, \pi_{y} \mid Y_{\mathrm{obs}}, \boldsymbol{z}, H_{0}\right) \\
\propto & {\left[\left(1-\pi_{x}\right)\left(1-\pi_{y}\right)\right]^{z_{1}}\left[\left(1-\pi_{x}\right) \pi_{y}\right]^{z_{2}} } \\
& \times\left[\pi_{x}\left(1-\pi_{y}\right)\right]^{z_{3}}\left(\pi_{x} \pi_{y}\right)^{z_{4}} \\
= & \pi_{x}^{z_{3}+z_{4}}\left(1-\pi_{x}\right)^{z_{1}+z_{2}} \times \pi_{y}^{z_{2}+z_{4}}\left(1-\pi_{y}\right)^{z_{1}+z_{3}} .
\end{aligned}
$$

Thus, the M-step is to calculate the complete-data MLEs of $\pi_{x}$ and $\pi_{y}$ as follows:

$$
\hat{\pi}_{0 x}=\frac{z_{3}+z_{4}}{z_{+}} \quad \text { and } \quad \hat{\pi}_{0 y}=\frac{z_{2}+z_{4}}{z_{+}},
$$

respectively. From (3.4), the E-step is to find the conditional expectations:

$$
E\left(z_{i} \mid Y_{\mathrm{obs}}, \hat{\boldsymbol{\pi}}_{0}\right)=\frac{n_{i} \hat{\pi}_{0 i} q}{p_{i}(1-q)+\hat{\pi}_{0 i} q}, \quad i=1, \ldots, m,
$$

where $\left\{\hat{\pi}_{0 i}\right\}_{i=1}^{4}$ are defined by (4.3). Alternatively, the chisquared statistic can be utilized to test $H_{0}$ against $H_{1}$. Let $\boldsymbol{p}=\left(p_{1}, \ldots, p_{4}\right)^{\top}$ and $\boldsymbol{\lambda}=\left(\lambda_{1}, \ldots, \lambda_{4}\right)^{\top}$, where $\lambda_{i}=(1-$ q) $p_{i}+q \pi_{i}$ for $i=1, \ldots, 4$. Then, we have $\boldsymbol{\lambda}=(1-q) \boldsymbol{p}+q \boldsymbol{\pi}$. Note that the restricted MLE $\hat{\boldsymbol{\pi}}_{0}=\left(\hat{\pi}_{01}, \ldots, \hat{\pi}_{04}\right)^{\top}$ of $\boldsymbol{\pi}$ under $H_{0}$ is given by (4.3), then

$$
\hat{\boldsymbol{\lambda}}_{0}=\left(\hat{\lambda}_{01}, \ldots, \hat{\lambda}_{04}\right)^{\top}=(1-q) \boldsymbol{p}+q \hat{\boldsymbol{\pi}}_{0}
$$

is the restricted MLE of $\boldsymbol{\lambda}$ under $H_{0}$. Therefore, under $H_{0}$, the chi-squared statistic

$$
\Lambda_{2}=\sum_{i=1}^{4} \frac{\left(n_{i}-n \hat{\lambda}_{0 i}\right)^{2}}{n \hat{\lambda}_{0 i}} \dot{\sim} \chi^{2}(1), \quad \text { as } n \rightarrow \infty .
$$

Table 3. Various values of $\pi_{1}$ and $\psi$ for the three scenarios specified by (4.7)

\begin{tabular}{l|cccccc}
\hline \hline & \multicolumn{7}{|c}{$\pi_{1}$} \\
\cline { 2 - 7 } & 0.200 & 0.300 & 0.500 & 0.700 & 0.800 & 0.900 \\
\hline Scenario 1: $\psi$ & 0.167 & 0.286 & 0.667 & 1.556 & 2.667 & 6.000 \\
Scenario 2: $\psi$ & 0.094 & 0.161 & 0.375 & 0.875 & 1.500 & 3.375 \\
Scenario 3: $\psi$ & 0.064 & 0.110 & 0.256 & 0.598 & 1.026 & 2.308 \\
\hline
\end{tabular}

\subsection{Comparison of the likelihood ratio test with the $\chi^{2}$ test}

For a given $\pi_{1}$, we consider the following three combinations of $\pi_{2}, \pi_{3}$ and $\pi_{4}$ such that $\sum_{i=1}^{4} \pi_{i}=1$ :

Scenario 1: $\quad\left(\pi_{2}, \pi_{3}, \pi_{4}\right)=(3,4,1) \frac{1-\pi_{1}}{8}, \psi=\frac{2 \pi_{1}}{3\left(1-\pi_{1}\right)}$;

Scenario 2: $\quad\left(\pi_{2}, \pi_{3}, \pi_{4}\right)=(4,10,1) \frac{1-\pi_{1}}{15}, \psi=\frac{3 \pi_{1}}{8\left(1-\pi_{1}\right)}$;

Scenario 3: $\left(\pi_{2}, \pi_{3}, \pi_{4}\right)=(6,13,1) \frac{1-\pi_{1}}{20}, \psi=\frac{10 \pi_{1}}{39\left(1-\pi_{1}\right)}$.

The sample sizes in simulations are designed by $n=$ 50(50)500. To compare the type I error rates (i.e., $\left.\pi_{1} \pi_{4} /\left(\pi_{2} \pi_{3}\right)=\psi=1\right)$, we take $\pi_{1}=\frac{3}{5}$ for scenario 1 , $\pi_{1}=\frac{8}{11}$ for scenario 2, and $\pi_{1}=\frac{39}{49}$ for scenario 3. For the comparison of powers (i.e., $\psi \neq 1$ ), the chosen $\pi_{1}$ and the corresponding $\psi$ are listed in Table 3. For a given pair $\left(n, \pi_{1}\right)$, we independently generate

$$
\begin{aligned}
& \left(n_{1}^{(l)}, \ldots, n_{4}^{(l)}\right) \sim \\
& \quad \operatorname{Multinomial}\left(n ; \frac{1}{8}+\frac{1}{2} \pi_{1}, \frac{1}{8}+\frac{1}{2} \pi_{2}, \frac{1}{8}+\frac{1}{2} \pi_{3}, \frac{1}{8}+\frac{1}{2} \pi_{4}\right)
\end{aligned}
$$

for $l=1, \ldots, L(L=1,000)$, where only $p_{i}=\frac{1}{4}(i=$ $1, \ldots, 4)$ and $q=\frac{1}{2}$ are considered. All hypothesis testings are conducted at level 0.05 . Let $r_{j}$ denote the number rejecting the null hypothesis (i.e., $H_{0}: \psi=1$ ) by the statistics $\Lambda_{j}(j=1,2)$. Hence, the actual significance level can be estimated by $r_{j} / L$ with $\psi=1$ and the power of the test statistic $\Lambda_{j}(j=1,2)$ can be estimated by $r_{j} / L$ with $\psi \neq 1$.

Figure 1 shows that some comparisons of type I error rates between the likelihood ratio test and the $\chi^{2}$ test for the three scenarios. In general, the chi-squared test has a better performance in controlling its Type I error rates around the pre-chosen nominal level than the likelihood ratio test, which can be seen in the three scenarios.

Figure 2 gives the comparisons of powers between the likelihood ratio test and the chi-squared test for different cases with $\psi \neq 1$. It is not difficult to find that there is no significant difference between the powers of the two test when $\psi$ is small (i.e., $<0.40$ ). When $0.60<\psi<1$, always 
(i) $\pi_{1}=3 / 5, \psi=1$ (Scenario 1 )

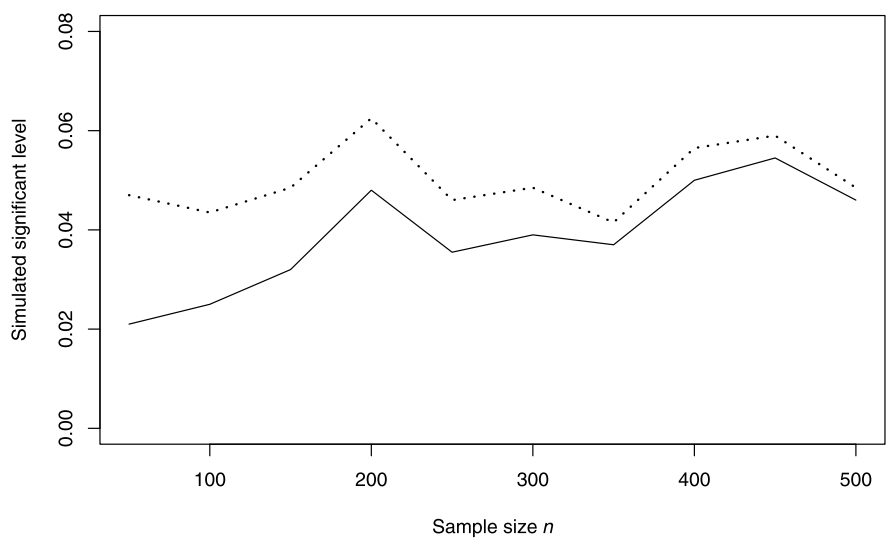

(ii) $\pi_{1}=8 / 11, \psi=1$ (Scenario 2 )

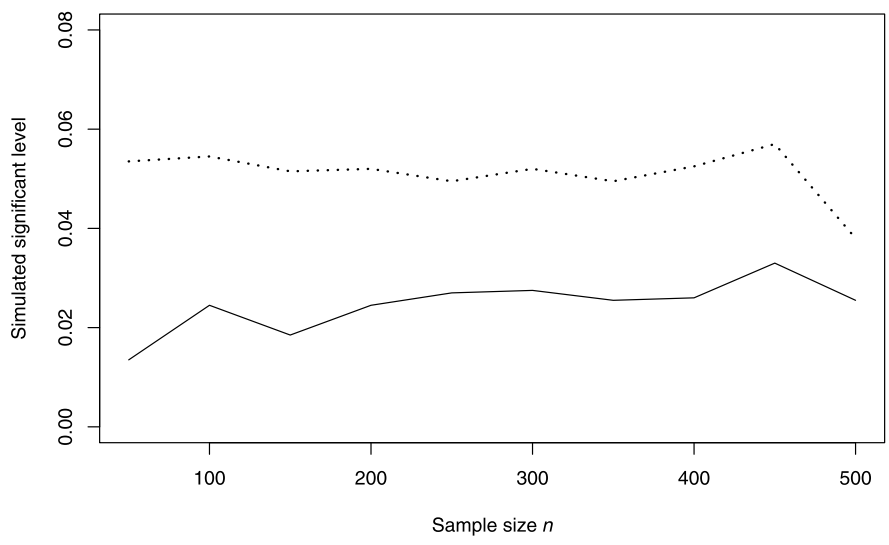

(iii) $\pi_{1}=39 / 49, \psi=1$ (Scenario 3 )

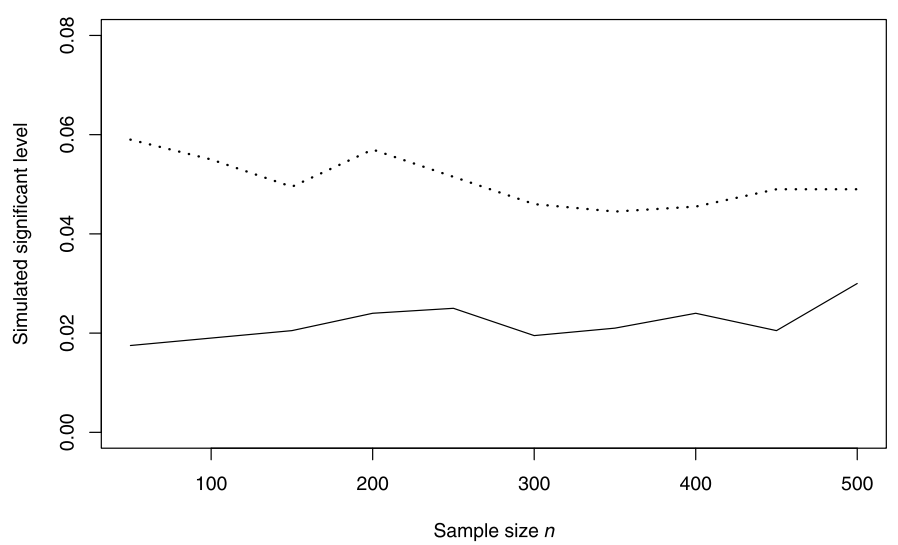

Figure 1. Comparisons of type I error rates between the likelihood ratio test (solid line) and the $\chi^{2}$ test (dotted line):

(a) $\pi_{1}=3 / 5, \psi=1$ (Scenario 1); (b) $\pi_{1}=8 / 11, \psi=1$ (Scenario 2); (c) $\pi_{1}=39 / 49, \psi=1$ (Scenario 3).

the likelihood ratio test is slightly less powerful than the chi-squared test, no matter whether the sample size is large or small.
Table 4. Questionnaire for the multi-category triangular model

\begin{tabular}{c|c|c|c|c}
\hline \hline Category & $U=1$ & $U=2$ & $\cdots$ & $U=m$ \\
\hline $1:\{Y=1\}$ & Block 1: & Block $2:_{-}$ & $\cdots$ & Block $m:-$ \\
\hline $2:\{Y=2\}$ & \multicolumn{4}{|c}{ Category 2: please put a tick in Block 2} \\
\hline$\vdots$ & \multicolumn{4}{|c}{$\cdots$} \\
\hline$m:\{Y=m\}$ & Category $m:$ please put a tick in Block $m$ \\
\hline Note: $\{Y=1\}$ represents the non-sensitive class.
\end{tabular}

\section{COMPARISON OF THE MULTI-CATEGORY PARALLEL MODEL WITH THE MULTI-CATEGORY TRIANGULAR MODEL}

In this section, we first briefly introduce the multicategory triangular model [11], and we then theoretically compare the efficiency of the multi-category parallel model with the multi-category triangular model by comparing the two variance-covariance matrices of the MLEs of parameters based on the trace criterion. Finally, we also consider the comparison of the degree of privacy protection for the two models.

\subsection{The survey design for the multi-category triangular model}

Tang et al. [11] proposed the survey design of the multicategory triangular model and developed the corresponding methods of statistical inference. Let $U$ and $Y$ be two independent categorical random variables as defined in Section 2, $\{Y=1\}$ denote the non-sensitive class and $\{Y=j\}$ the sensitive classes for $j=2, \ldots, m$. Define $p_{j}=\operatorname{Pr}\{U=j\}$ and $\pi_{j}=\operatorname{Pr}\{Y=j\}, j=1, \ldots, m$. Assume that $\left\{p_{j}\right\}_{j=1}^{m}$ are known. The objective is to estimate $\boldsymbol{\pi}=\left(\pi_{1}, \ldots, \pi_{m}\right)^{\top} \in$ $\mathbb{T}_{m}$. The survey design shown in Table 4 can be described as following: Since the category 1 (i.e., $\{Y=1\}$ ) is a nonsensitive class, it is supposed that the respondents belonging to this class can provide correct answers (i.e., putting a tick in Block $j$ for $j=1, \ldots, m$ ) according to their true status. In addition, the respondents belonging to the category $j(j=2, \ldots, m)$ will be asked to put a tick in Block $j$. The cell probabilities $\left\{\pi_{j}\right\}$, the observed frequencies $\left\{n_{j}\right\}$ and the unobservable frequencies $\left\{z_{j}\right\}$ are shown in Table 5 .

\subsection{The difference between two traces of variance-covariance matrice of the MLEs of parameters}

(a) The variance-covariance matrix of $\hat{\boldsymbol{\pi}}_{\mathrm{MT}}$

For the multi-category triangular model, let $\boldsymbol{\theta}=$ $\left(\theta_{1}, \ldots, \theta_{m}\right)^{\top}$, where $\theta_{1}=p_{1} \pi_{1}$ and $\theta_{j}=p_{j} \pi_{1}+\pi_{j}(j=$ $2, \ldots, m)$ represent the proportions that the respondents belonging to Block $j$. In matrix notation, we have 



(b1) $\psi=0.286$ (Scenario 1)

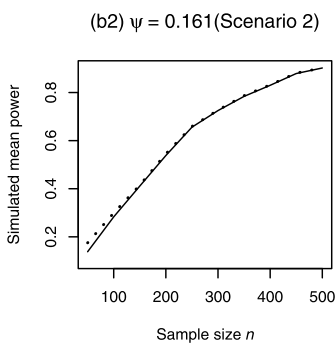

(b3) $\psi=0.110$ (Scenario 3)
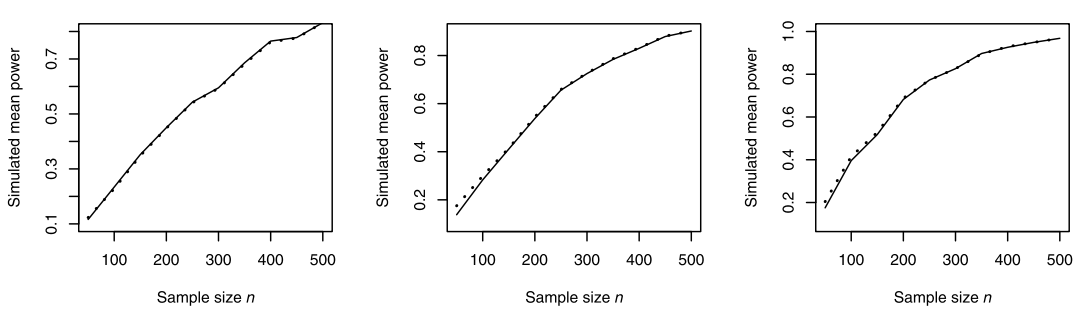

(c1) $\psi=0.667$ (Scenario 1)


(c3) $\psi=0.256$ (Scenario 3)

Sample size $n$

Sample size $n$

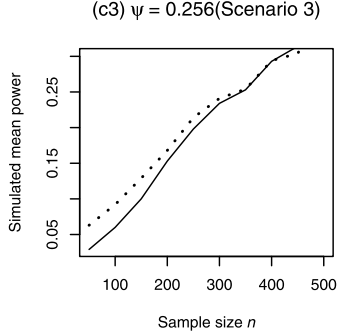

(d1) $\psi=1.556$ (Scenario 1)

(d2) $\psi=0.875$ (Scenario 2)

(d3) $\psi=0.598$ (Scenario 3)
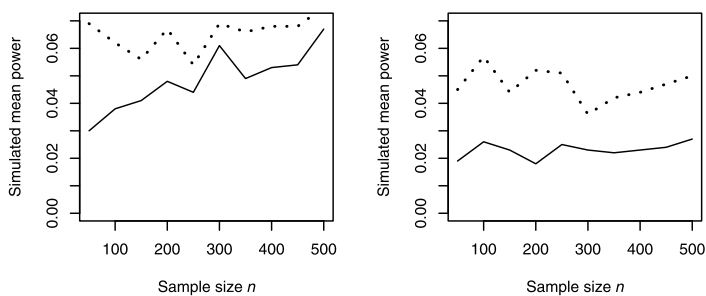

Sample size $n$

(e1) $\psi=2.667$ (Scenario 1)

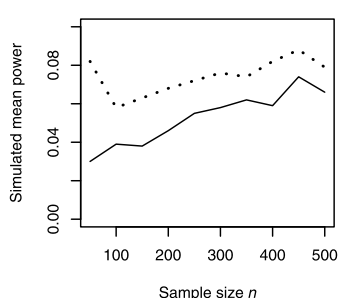

(e2) $\psi=1.500$ (Scenario 2)
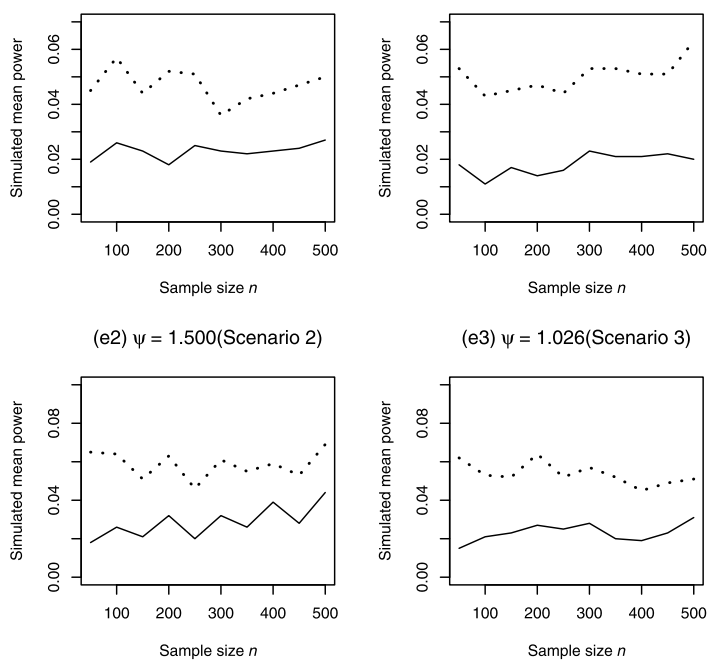

Sample size $n$

(e3) $\psi=1.026$ (Scenario 3)



(f1) $\psi=6.000$ (Scenario 1)

(†2) $\psi=3.3755$ (Scenario 2)
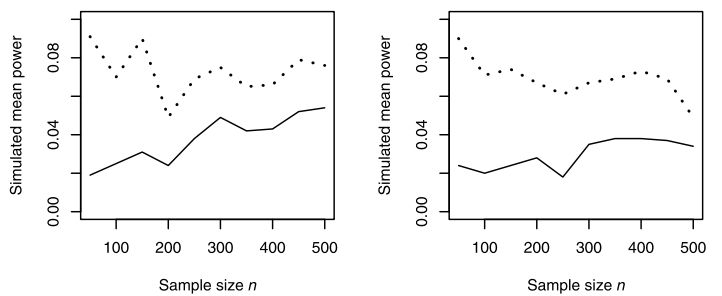

(f3) $\psi=2.308$ (Scenario 3)

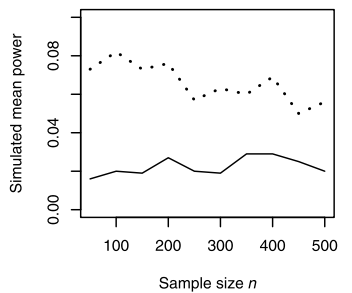

Figure 2. Comparisons of powers between the likelihood ratio test (solid line) and the $\chi^{2}$ test (dotted line).

$144 Y$. Liu and G. Tian 
Table 5. Questionnaire for the multi-category triangular model

\begin{tabular}{|c|c|c|c|c|c|}
\hline Category & $U=1$ & $U=2$ & $\cdots$ & $U=m$ & Total \\
\hline $1: Y=1$ & $p_{1} \pi_{1}$ & $p_{2} \pi_{1}$ & $\cdots$ & $p_{m} \pi_{1}$ & $\pi_{1}\left(z_{1}\right)$ \\
\hline $2: Y=2$ & & & & & $\pi_{2}\left(z_{2}\right)$ \\
\hline : & & & & &  \\
\hline$m: Y=m$ & & & & & $\overline{\pi_{m}\left(z_{m}\right)}$ \\
\hline Total & $p_{1} \pi_{1}\left(n_{1}\right)$ & $\begin{array}{l}p_{2} \pi_{1}+ \\
\pi_{2}\left(n_{2}\right)\end{array}$ & $\cdots$ & $\begin{array}{l}p_{m} \pi_{1}+ \\
\pi_{m}\left(n_{m}\right)\end{array}$ & $1(n)$ \\
\hline
\end{tabular}

Note: $n=\sum_{j=1}^{m} n_{j}, z_{1}=n-\sum_{j=2}^{m} z_{j}$, where $\left\{z_{2}, \ldots, z_{m}\right\}$ are unobservable.

$$
\boldsymbol{\theta}=\mathbf{P} \boldsymbol{\pi}=\left(\begin{array}{cc}
p_{1} & 0_{m-1}^{\top} \\
\boldsymbol{p}_{-1} & \mathbf{I}_{m-1}
\end{array}\right)\left(\begin{array}{c}
\pi_{1} \\
\vdots \\
\pi_{m}
\end{array}\right)
$$

where $\boldsymbol{p}_{-1}=\left(p_{2}, \ldots, p_{m}\right)^{\top}, \mathbf{0}_{m-1}$ is the $(m-1) \times 1$ vector of zeros and $\mathbf{I}_{m-1}$ denotes the $(m-1) \times(m-1)$ identity matrix. Since $\left(n_{1}, \ldots, n_{m}\right)^{\top} \sim \operatorname{Multinomial}\left(n ; \theta_{1}, \ldots, \theta_{m}\right)$, the MLE of $\theta_{j}$ is $\hat{\theta}_{j}=n_{j} / n$. Thus, the variance-covariance matrix of $\hat{\boldsymbol{\theta}}=\left(\hat{\theta}_{1}, \ldots, \hat{\theta}_{m}\right)^{\top}$ is

$$
\operatorname{Var}(\hat{\boldsymbol{\theta}})=\frac{1}{n}\left[\operatorname{diag}(\boldsymbol{\theta})-\boldsymbol{\theta} \boldsymbol{\theta}^{\top}\right] .
$$

In general, the MLE $\hat{\boldsymbol{\pi}}_{\mathrm{MT}}$ of $\boldsymbol{\pi}$ for the multi-category triangular model can be obtained by using the EM algorithm [11]. However, for some cases, we can obtain a closedform solution to $\hat{\boldsymbol{\pi}}_{\mathrm{MT}}$. In fact, from (5.1), we have $\boldsymbol{\pi}=\mathbf{P}^{-1} \boldsymbol{\theta}$. Since the MLE of $\boldsymbol{\theta}$ is $\hat{\boldsymbol{\theta}}=\left(n_{1} / n, \ldots, n_{m} / n\right)^{\top}$, an alternative estimator of $\boldsymbol{\pi}$ for the multi-category triangular model is given by

$$
\hat{\boldsymbol{\pi}}_{v}=\mathbf{P}^{-1} \hat{\boldsymbol{\theta}}=\left(\begin{array}{cc}
1 / p_{1} & \mathbf{0}_{m-1}^{\top} \\
-\boldsymbol{p}_{-1} / p_{1} & \mathbf{I}_{m-1}
\end{array}\right)\left(\begin{array}{c}
n_{1} / n \\
\vdots \\
n_{m} / n
\end{array}\right) .
$$

It should be noted that it is possible that $\hat{\boldsymbol{\pi}}_{v} \notin \mathbb{T}_{m}$. For example, let $m=4, p_{1}=\cdots=p_{4}=0.25$ and $\left(n_{1}, \ldots, n_{4}\right)^{\top}=$ $(12,8,6,19)^{\top}$, then

$$
\hat{\boldsymbol{\pi}}_{v}=(1.066667,-0.088889,-0.133333,0.155556)^{\top} \notin \mathbb{T}_{4} .
$$

In this paper, the estimator $\hat{\boldsymbol{\pi}}_{v}$ given by (5.3) is said to be valid if $\hat{\boldsymbol{\pi}}_{v} \in \mathbb{T}_{m}$. Clearly, if $\hat{\boldsymbol{\pi}}_{v}$ specified by (5.3) is a valid estimator of $\boldsymbol{\pi}$ then $\hat{\boldsymbol{\pi}}_{v}=\hat{\boldsymbol{\pi}}_{\mathrm{MT}}$. In the following discussion, we only consider the case of valid estimators.

Hence, from (5.3), (5.2) and (5.1), the variance-covariance matrix of $\hat{\boldsymbol{\pi}}_{\mathrm{MT}}$ is

$$
\begin{aligned}
\operatorname{Var}\left(\hat{\boldsymbol{\pi}}_{\mathrm{MT}}\right) & =\operatorname{Var}\left(\hat{\boldsymbol{\pi}}_{v}\right) \\
& =\mathbf{P}^{-1} \operatorname{Var}(\hat{\boldsymbol{\theta}})\left(\mathbf{P}^{-1}\right)^{\top} \\
& =\frac{1}{n}\left[\mathbf{P}^{-1} \operatorname{diag}(\mathbf{P} \boldsymbol{\pi})\left(\mathbf{P}^{-1}\right)^{\top}-\boldsymbol{\pi} \boldsymbol{\pi}^{\top}\right],
\end{aligned}
$$

or equivalently

$$
\begin{aligned}
\operatorname{Var}\left(\hat{\pi}_{\mathrm{MT} 1}\right) & =\frac{1}{n}\left(\frac{\pi_{1}}{p_{1}}-\pi_{1}^{2}\right), \\
\operatorname{Var}\left(\hat{\pi}_{\mathrm{MT} j}\right) & =\frac{1}{n}\left(\frac{p_{j}^{2}}{p_{1}} \pi_{1}+p_{j} \pi_{1}+\pi_{j}-\pi_{j}^{2}\right), \\
\operatorname{Cov}\left(\hat{\pi}_{\mathrm{MT} 1}, \hat{\pi}_{\mathrm{MT} j}\right) & =\frac{1}{n}\left(-\frac{p_{j}}{p_{1}} \pi_{1}-\pi_{1} \pi_{j}\right), \quad \text { and } \\
\operatorname{Cov}\left(\hat{\pi}_{\mathrm{MT} i}, \hat{\pi}_{\mathrm{MT} j}\right) & =\frac{1}{n}\left(\frac{p_{i} p_{j}}{p_{1}} \pi_{1}-\pi_{i} \pi_{j}\right),
\end{aligned}
$$

where $i \neq j, i, j=2, \ldots, m$.

(b) The comparison between $\operatorname{Var}\left(\hat{\pi}_{\mathrm{MT}}\right)$ and $\operatorname{Var}\left(\hat{\boldsymbol{\pi}}_{\mathrm{MP}}\right)$

In the multi-category triangular model, there are only two parameter vectors (i.e., $\boldsymbol{\pi}$ and $\boldsymbol{p}$ ), while in the multi-category parallel model, besides $\boldsymbol{\pi}$ and $\boldsymbol{p}$, there is an additional parameter $q$. By controlling $q$ within a certain subset of the unit interval, we may have $\operatorname{Var}\left(\hat{\boldsymbol{\pi}}_{\mathrm{MP}}\right)$ being 'smaller' than $\operatorname{Var}\left(\hat{\boldsymbol{\pi}}_{\mathrm{MT}}\right)$. In the follows, we only apply the trace criterion in the comparison between $\operatorname{Var}\left(\hat{\boldsymbol{\pi}}_{\mathrm{MT}}\right)$ and $\operatorname{Var}\left(\hat{\boldsymbol{\pi}}_{\mathrm{MP}}\right)$.

First, from (5.4) and (5.5), we have

$$
\begin{aligned}
& \operatorname{tr}\left[\operatorname{Var}\left(\hat{\boldsymbol{\pi}}_{\mathrm{MT}}\right)\right] \\
&=\frac{1}{n}\left[\frac{\pi_{1}}{p_{1}}-\pi_{1}+\pi_{1}\left(1-\pi_{1}\right)\right. \\
&\left.+\sum_{j=2}^{m}\left(\frac{p_{j}^{2}}{p_{1}} \pi_{1}+p_{j} \pi_{1}+\pi_{j}\left(1-\pi_{j}\right)\right)\right] \\
&= \frac{\pi_{1}}{n}\left(\frac{1}{p_{1}}-p_{1}+\sum_{j=2}^{m} \frac{p_{j}^{2}}{p_{1}}\right)+\frac{1}{n} \sum_{j=1}^{m} \pi_{j}\left(1-\pi_{j}\right) .
\end{aligned}
$$

Next, from (3.12) and (3.10), we obtain

$$
\begin{aligned}
\operatorname{tr}\left[\operatorname{Var}\left(\hat{\boldsymbol{\pi}}_{\mathrm{MP}}\right)\right] \\
=\frac{1}{n q^{2}} \sum_{j=1}^{m} \lambda_{j}\left(1-\lambda_{j}\right) \\
=\frac{1}{n q^{2}} \sum_{j=1}^{m}\left[p_{j}(1-q)+\pi_{j} q\right]\left[1-p_{j}(1-q)-\pi_{j} q\right] \\
=\frac{1-q}{n q^{2}}\left(1+q-2 q \sum_{j=1}^{m} \pi_{j} p_{j}-(1-q) \sum_{j=1}^{m} p_{j}^{2}\right) \\
\quad+\frac{1}{n} \sum_{j=1}^{m} \pi_{j}\left(1-\pi_{j}\right) .
\end{aligned}
$$

Thus, the difference of them is

$$
\begin{array}{r}
\operatorname{tr}\left[\operatorname{Var}\left(\hat{\boldsymbol{\pi}}_{\mathrm{MT}}\right)\right]-\operatorname{tr}\left[\operatorname{Var}\left(\hat{\boldsymbol{\pi}}_{\mathrm{MP}}\right)\right] \\
=\frac{\pi_{1}}{n}\left(\frac{1}{p_{1}}-p_{1}+\sum_{j=2}^{m} \frac{p_{j}^{2}}{p_{1}}\right)
\end{array}
$$




$$
\begin{aligned}
& -\frac{1-q}{n q^{2}}\left(1+q-2 q \sum_{j=1}^{m} \pi_{j} p_{j}-(1-q) \sum_{j=1}^{m} p_{j}^{2}\right) \\
= & \frac{1}{n q^{2}} h(q \mid \boldsymbol{\pi}, \boldsymbol{p}),
\end{aligned}
$$

where

$$
\begin{aligned}
h(q \mid \boldsymbol{\pi}, \boldsymbol{p})= & {\left[\pi_{1}\left(\frac{1}{p_{1}}-p_{1}+\sum_{j=2}^{m} \frac{p_{j}^{2}}{p_{1}}\right)\right.} \\
& \left.+\left(1-2 \sum_{j=1}^{m} \pi_{j} p_{j}+\sum_{j=1}^{m} p_{j}^{2}\right)\right] q^{2} \\
& +2\left(\sum_{j=1}^{m} \pi_{j} p_{j}-\sum_{j=1}^{m} p_{j}^{2}\right) q-1+\sum_{j=1}^{m} p_{j}^{2} \\
\hat{=} & a q^{2}+b q+c
\end{aligned}
$$

is a quadratic function of $q$ for given $\boldsymbol{\pi}$ and $\boldsymbol{p}$. In both survey designs (see Table 1 and Table 4 ), we require $p_{1} \in(0,1)$ so that $1-p_{1}^{2}>0$. In addition, $0 \leq \sum_{j=1}^{m} \pi_{j}^{2} \leq \sum_{j=1}^{m} \pi_{j}=1$. Thus,

$$
\begin{aligned}
a & =\pi_{1}\left(\frac{1}{p_{1}}-p_{1}+\sum_{j=2}^{m} \frac{p_{j}^{2}}{p_{1}}\right)+\left(1-2 \sum_{j=1}^{m} \pi_{j} p_{j}+\sum_{j=1}^{m} p_{j}^{2}\right) \\
& =\frac{\pi_{1}}{p_{1}}\left(1-p_{1}^{2}+\sum_{j=2}^{m} p_{j}^{2}\right)+1-\sum_{j=1}^{m} \pi_{j}^{2}+\sum_{j=1}^{m}\left(\pi_{j}-p_{j}\right)^{2}
\end{aligned}
$$$$
>0 \text {. }
$$

Now, the discriminant of the quadratic function $h(q \mid \boldsymbol{\pi}, \boldsymbol{p})$ is

$$
\begin{aligned}
D(h)= & b^{2}-4 a c \\
= & 4\left(\sum_{j=1}^{m} \pi_{j} p_{j}-\sum_{j=1}^{m} p_{j}^{2}\right)^{2} \\
& +4 \pi_{1}\left(\frac{1}{p_{1}}-p_{1}+\sum_{j=2}^{m} \frac{p_{j}^{2}}{p_{1}}\right)\left(1-\sum_{j=1}^{m} p_{j}^{2}\right) \\
& +4\left(1-2 \sum_{j=1}^{m} \pi_{j} p_{j}+\sum_{j=1}^{m} p_{j}^{2}\right)\left(1-\sum_{j=1}^{m} p_{j}^{2}\right) \\
= & 4\left(1-\sum_{j=1}^{m} \pi_{j} p_{j}\right)^{2} \\
& +\frac{4 \pi_{1}}{p_{1}}\left(1-p_{1}^{2}+\sum_{j=2}^{m} p_{j}^{2}\right)\left(1-\sum_{j=1}^{m} p_{j}^{2}\right) \\
> & 0 .
\end{aligned}
$$

By applying Result (iii) of the following lemma,

Lemma 1. Let $a>0$ and $D(f)=b^{2}-4 a c$ denote the discriminant of a parabola $f(x)=a x^{2}+b x+c$. We have

(i) If $D(f)<0$, then $f(x)>0$ for all $x \in(-\infty, \infty)$.

146 Y. Liu and G. Tian (ii) If $D(f)=0$, then $f(x) \geq 0$ for all $x \in(-\infty, \infty)$, and $f(x)$ reaches its minimum zero at $x=-b /(2 a)$.

(iii) If $D(f)>0$, then $f(x)>0$ for all $x \in\left(-\infty, x_{1}\right) \cup$ $\left(x_{2}, \infty\right)$ and $f(x) \leq 0$ for all $x \in\left[x_{1}, x_{2}\right]$, where $x_{1}=$ $[-b-\sqrt{D(f)}] /(2 a)$ and $x_{2}=[-b+\sqrt{D(f)}] /(2 a)$.

We immediately obtain the following theorem.

Theorem 2. Let $\boldsymbol{\pi} \in \mathbb{T}_{m}$ and $\boldsymbol{p} \in \mathbb{T}_{m}$, we always have $\operatorname{athrmtr}\left[\operatorname{Var}\left(\hat{\boldsymbol{\pi}}_{\mathrm{MT}}\right)\right]>\operatorname{tr}\left[\operatorname{Var}\left(\hat{\boldsymbol{\pi}}_{\mathrm{MP}}\right)\right]$ for any $q \in\left(0, q_{\mathrm{L}}\right) \cup$ $\left(q_{\mathrm{U}}, 1\right)$, where

$$
\begin{aligned}
& q_{\mathrm{L}}=\max \left\{0, \frac{-b-\sqrt{b^{2}-4 a c}}{2 a}\right\} \quad \text { and } \\
& q_{\mathrm{U}}=\min \left\{1, \frac{-b+\sqrt{b^{2}-4 a c}}{2 a}\right\},
\end{aligned}
$$

where $a, b$ and $c$ are defined in (5.6).

\subsection{Degree of privacy protection}

In this subsection, we compare degrees of privacy protection (DPP) of the multi-category parallel model with those of the multi-category triangular model. For the multicategory parallel model (see Table 1), we define

$$
\begin{aligned}
\operatorname{DPP}_{\mathrm{MP}}\left(\pi_{1}, p_{1}, q\right) & =\operatorname{Pr}(Y=1 \mid \text { two circles are connected }), \\
\operatorname{DPP}_{\mathrm{MP}}\left(\pi_{2}, p_{2}, q\right) & =\operatorname{Pr}(Y=2 \mid \text { two triangles are connected }), \\
& \vdots \\
\operatorname{DPP}_{\mathrm{MP}}\left(\pi_{m}, p_{m}, q\right) & =\operatorname{Pr}(Y=m \mid \text { two dots are connected }),
\end{aligned}
$$

where, for example, $\operatorname{DPP}_{\mathrm{MP}}\left(\pi_{1}, p_{1}, q\right)$ denotes the conditional probability that the respondent belongs to the subclass $\{Y=1\}$ given that he/she connected the two circles. For the multi-category triangular model (see Table 4), we can similarly define

$$
\begin{aligned}
\operatorname{DPP}_{\mathrm{MT}}\left(\pi_{1}, p_{1}\right) & =\operatorname{Pr}(Y=1 \mid \text { a tick is put in Block } 1), \\
\operatorname{DPP}_{\mathrm{MT}}\left(\pi_{2}, p_{2}\right) & =\operatorname{Pr}(Y=2 \mid \text { a tick is put in Block } 2), \\
& \vdots \\
\operatorname{DPP}_{\mathrm{MT}}\left(\pi_{m}, p_{m}\right) & =\operatorname{Pr}(Y=m \mid \text { a tick is put in Block } m) .
\end{aligned}
$$

First, for any $q \in(0,1), \boldsymbol{\pi} \in \mathbb{T}_{m}$ and $\boldsymbol{p} \in \mathbb{T}_{m}$, we always have

$\operatorname{DPP}_{\mathrm{MT}}\left(\pi_{1}, p_{1}\right)=1>\frac{\pi_{1} q}{p_{1}(1-q)+\pi_{1} q}=\operatorname{DPP}_{\mathrm{MP}}\left(\pi_{1}, p_{1}, q\right)$.

Next, when $0<q<\frac{1}{1+\pi_{1}}$, we obtain

$$
\begin{aligned}
\operatorname{DPP}_{\mathrm{MT}}\left(\pi_{j}, p_{j}\right) & =\frac{\pi_{j}}{p_{j} \pi_{1}+\pi_{j}} \\
& >\frac{\pi_{j} q}{p_{j}(1-q)+\pi_{j} q} \\
& =\operatorname{DPP}_{\mathrm{MP}}\left(\pi_{j}, p_{j}, q\right), \quad j=2, \ldots, m,
\end{aligned}
$$


Table 6. Survey data from Williamson and Haber (1994)

\begin{tabular}{l|l|l|c}
\hline \hline \multirow{2}{*}{$\begin{array}{l}\text { Number of } \\
\text { sex partners }\end{array}$} & $Y=0($ low $)$ & $Y=1($ high $)$ & Missing \\
\cline { 2 - 4 }$X=0(0-3)$ & $144\left(m_{1}, \pi_{1}\right)$ & $123\left(m_{2}, \pi_{2}\right)$ & 17 \\
$X=1(\geq 4)$ & $237\left(m_{3}, \pi_{3}\right)$ & $148\left(m_{4}, \pi_{4}\right)$ & 17 \\
\hline
\end{tabular}

for any $\boldsymbol{\pi} \in \mathbb{T}_{m}$ and $\boldsymbol{p} \in \mathbb{T}_{m}$. Inequalities (5.7) and (5.8) show that if we choose $q$ within the open interval $\left(0, \frac{1}{1+\pi_{1}}\right)$, the multi-category parallel model is more efficient than the multi-category triangular model in protecting the individual's privacy for any $\boldsymbol{\pi} \in \mathbb{T}_{m}$ and $\boldsymbol{p} \in \mathbb{T}_{m}$.

Williamson and Haber [16] reported a study aimed to examine the relationship among disease status of cervical cancer, the number of sexual partners and income. Respondents were women of 20-79 year old in Fulton or Dekalb County in Atlanta, Georgia. Table 6 displays the cross-classification of income (low or high, denoted by $Y=0$ or $Y=1$ ) and number of sex partners ('few' (0-3) or 'many' $(\geq 4)$, denoted by $X=0$ or $X=1$ ). Since all four questions (i.e., the number of sex partners and income status) are highly sensitive to respondents, a sizable proportion (19.9\% in this example) of the responses would be missing because of 'unknown' or 'refused to answer' in a telephone interview. The major objective is to examine if association exists between the number of sex partners and income. The existing multicategory triangular model and the corresponding statistical methods [11] cannot be applied to such studies because each of the four subclasses $\{X=0, Y=0\},\{X=0, Y=1\}$, $\{X=1, Y=0\}$ and $\{X=1, Y=1\}$ is sensitive to respondents. To demonstrate the proposed multi-category parallel design in Tables 1 and 2 and the developed estimation methods in Sections 3 and 4 , we let $m=4$ and define $W=0$ if the respondent's birthday is in the first half of a month and $W=1$ otherwise. Similarly, we define $U=i$ if the respondent was born in the $i$-th quarter of a year $(i=1, \ldots, 4)$. Thus, it is reasonable to assume that $q=\operatorname{Pr}(W=1)=0.5$ and $p_{i}=\operatorname{Pr}(U=i)=0.25$ for each $i$.

\section{AN EXAMPLE}

To obtain the observed data $Y_{\text {obs }}=\left\{n ; n_{1}, \ldots, n_{4}\right\}$ in the four-category parallel model (see Table 2), we only consider the complete observations in Table 6 and discard the associated missing data and obtain $n=m_{1}+\cdots+m_{4}=$ $144+123+237+148=652$. Note that $n_{1}$ denotes the number of respondents connecting the two circles in Table 2, $n_{2}$ is the number of respondents connecting the two triangles, $n_{3}$ is the number of respondents connecting the two rectangles, and $n_{4}$ is the number of respondents connecting the two dots. Let $z_{1}, z_{2}, z_{3}$ and $z_{4}$ denote the number of respondents belonging to $\{X=0, Y=0\} \cap\{W=1\}$, $\{X=0, Y=1\} \cap\{W=1\},\{X=1, Y=0\} \cap\{W=1\}$ and $\{X=1, Y=1\} \cap\{W=1\}$ in Table 2 respectively. Since $q=1 / 2$, we have $z_{i}=m_{i} / 2$ for the ideal situation, i.e., $\left(z_{1}, z_{2}, z_{3}, z_{4}\right)^{\top} \approx(72,62,118,74)^{\top}$. Furthermore, let $n_{i}^{\prime}$ denote the number of respondents belonging to $\{U=i\} \cap\{W=0\}$ for $i=1, \ldots, 4$ in Table 2. To obtain these $\left\{n_{i}^{\prime}\right\}_{i=1}^{4}$ by considering the sampling error, we first generate 50 i.i.d. samples from

$$
\begin{aligned}
& \operatorname{Multinomial}\left(n-\sum_{i=1}^{4} z_{i} ; p_{1}, p_{2}, p_{3}, p_{4}\right) \\
& =\operatorname{Multinomial}\left(326 ; 0.25 \times \mathbf{1}_{4}\right)
\end{aligned}
$$

and then average these counts for each component, yielding $\left(n_{1}^{\prime}, n_{2}^{\prime}, n_{3}^{\prime}, n_{4}^{\prime}\right)^{\top}=(81,82,81,82)^{\top}$. Therefore, we obtain the following observed counts

$$
\begin{aligned}
& \left(n_{1}, n_{2}, n_{3}, n_{4}\right)^{\top} \\
& \quad=\left(z_{1}+n_{1}^{\prime}, z_{2}+n_{2}^{\prime}, z_{3}+n_{3}^{\prime}, z_{4}+n_{4}^{\prime}\right)^{\top} \\
& \quad=(153,144,199,156)^{\top} .
\end{aligned}
$$

Using $\boldsymbol{\pi}^{(0)}=0.25 \times \mathbf{1}_{4}$ as the initial values, the EM algorithm in (3.3) and (3.5) converged in 25 iterations. The resultant MLEs for $\boldsymbol{\pi}=\left(\pi_{1}, \ldots, \pi_{4}\right)^{\top}$ and the odds ratio $\psi$ are listed in the second column of Table 7. Based on (3.6), we generate $G=10,000$ bootstrap samples to estimate the standard errors of $\left\{\hat{\pi}_{\mathrm{MP} i}\right\}_{i=1}^{4}$ and $\hat{\psi}$, which are given in the third column of Table 7 . The corresponding 95\% normalbased bootstrap CIs and non-normal-based bootstrap CIs are displayed in the fourth and the fifth columns of Table 7 . Since the two bootstrap CIs of $\psi$ include 1 , we do not have reason to believe that there exists association between the number of sex partners and income. According to (3.9), we obtain $\hat{\pi}_{v}=(0.2193252,0.1917178,0.3604294,0.2285276)^{\top}$. Since $\hat{\boldsymbol{\pi}}_{v} \in \mathbb{T}_{4}$, we know that $\hat{\boldsymbol{\pi}}_{v}$ is a valid estimator of $\boldsymbol{\pi}$ and $\hat{\boldsymbol{\pi}}_{v}=\hat{\boldsymbol{\pi}}_{\mathrm{MP}}$. Based on (3.12), the estimated variancecovariance matrix of $\hat{\boldsymbol{\pi}}_{\mathrm{MP}}$ is

$$
\begin{aligned}
& \widehat{\operatorname{Var}}\left(\hat{\boldsymbol{\pi}}_{\mathrm{MP}}\right) \\
& \quad=\left(\begin{array}{rrrr}
0.00110 & -0.00032 & -0.00044 & -0.00034 \\
-0.00032 & 0.00106 & -0.00041 & -0.00032 \\
-0.00044 & -0.00041 & 0.00130 & -0.00045 \\
-0.00034 & -0.00032 & -0.00045 & 0.00112
\end{array}\right)
\end{aligned}
$$

so that the unbiased estimates of $\left\{\operatorname{Var}\left(\hat{\pi}_{\mathrm{MP} i}\right)\right\}_{i=1}^{4}$, from (3.13), are given by

$$
\begin{aligned}
& \left(\widehat{\operatorname{Var}}\left(\hat{\pi}_{\mathrm{MP} 1}\right), \ldots, \widehat{\operatorname{Var}}\left(\hat{\pi}_{\mathrm{MP} 4}\right)\right)^{\top} \\
& \quad=\left(\begin{array}{ll}
0.00110,0.00106,0.00130, & 0.00112
\end{array}\right)^{\top} .
\end{aligned}
$$

Therefore, from (3.14), (3.16) and (3.18), the 95\% Wald, Wilson and likelihood ratio CIs of $\left\{\pi_{i}\right\}_{i=1}^{4}$ can be calculated and are given in the second, the fourth and sixth columns of Table 8. We noted that the width of the $95 \%$ Wilson CI of $\pi_{i}$ is slightly shorter than those of the $95 \%$ Wald CIs and LRCIs of $\pi_{i}$. 
Table 7. MLEs and two bootstrap Cls of parameters for the observed counts $\left(n_{1}, n_{2}, n_{3}, n_{4}\right)^{\top}=(153,144,199,156)^{\top}$

\begin{tabular}{|c|c|c|c|c|}
\hline Parameter & std & & $95 \%$ bootstrap $\mathrm{CI}^{\dagger}$ & $95 \%$ bootstrap $\mathrm{CI}^{\ddagger}$ \\
\hline$\pi_{1}$ & 0.2196 & 0.0328 & {$[0.1553,0.2839]$} & {$[0.1549,0.2837]$} \\
\hline$\pi_{2}$ & 0.1919 & 0.0327 & {$[0.1277,0.2561]$} & {$[0.1273,0.2561]$} \\
\hline$\pi_{3}$ & 0.3604 & 0.0359 & {$[0.2900,0.4308]$} & {$[0.2929,0.4310]$} \\
\hline$\pi_{4}$ & 0.2281 & 0.0338 & {$[0.1619,0.2943]$} & {$[0.1641,0.2960]$} \\
\hline$\psi$ & 0.7661 & 0.2712 & {$[0.2344,1.2977]$} & {$[0.3690,1.4099]$} \\
\hline
\end{tabular}

$\mathrm{CI}^{\dagger}$ : Normal-based bootstrap CIs, cf. (3.7). CI ${ }^{\ddagger}$ : Non-normal-based bootstrap CIs, cf. (3.8).

Table 8. Three asymptotic $95 \% \mathrm{Cls}$ of parameters for large sample sizes

\begin{tabular}{c|c|c|c|c|c|c}
\hline \hline Parameter & Wald CI & Width & Wilson CI & Width & LRCI & Width \\
\hline$\pi_{1}$ & {$[0.1542,0.2844]$} & 0.1302 & {$[0.1575,0.2874]$} & 0.1299 & {$[0.1564,0.2864]$} & 0.1300 \\
$\pi_{2}$ & {$[0.1280,0.2554]$} & 0.1274 & {$[0.1314,0.2586]$} & 0.1272 & {$[0.1303,0.2575]$} & 0.1272 \\
$\pi_{3}$ & {$[0.2897,0.4312]$} & 0.1415 & {$[0.2922,0.4332]$} & 0.1410 & {$[0.2914,0.4326]$} \\
$\pi_{4}$ & {$[0.1630,0.2941]$} & 0.1311 & {$[0.1662,0.2970]$} & 0.1308 & {$[0.1652,0.2960]$} & 0.1412 \\
\hline
\end{tabular}

\section{DISCUSSION}

As a natural generalization of the NRR parallel model of Tian [12], we develop an NRR multi-category parallel model for a single sensitive question with multiple answers/outcomes. When comparing with the existing NRR multi-category triangular model, the newly developed model has several significant advantages: (i) A wider applicability. The multi-category parallel model can be applied to such situations where all population subclasses could be sensitive, while the former is only applicable in the case that at least one of the population subclasses is non-sensitive. (ii) A higher efficiency. Because of the introduction of additional parameter $q=\operatorname{Pr}(W=1) \in(0,1)$, the multi-category parallel model is more efficient than the multi-category triangular model for a certain range of $q$ (see Theorem 2 in Section 5.2). (iii) A better degree of privacy protection. The comparisons in Section 5.3 show that if $0<q<\frac{1}{1+\pi_{1}}$, the multi-category parallel model is more efficient than the multi-category triangular model in protecting the individual's privacy for any $\boldsymbol{\pi} \in \mathbb{T}_{m}$ and $\boldsymbol{p} \in \mathbb{T}_{m}$.

How to choose the two non-sensitive variables $W$ and $U$ in Table 1 is an important issue in practice. On the one hand, since $W$ is a binary variable, we could define $W=0$ if the respondent was born between January and June; or the respondent was born in an odd numbered month; or the respondent's birthday is in the first half of the month; or the respondent's age is odd numbered; or the respondent's house/apartment number is even. On the other hand, since $U$ is an $m$-category variable, for example, when $m=3$, we may let

$$
\begin{aligned}
& U=1 \quad \begin{array}{l}
\text { if the respondent's mother was born in } \\
\text { January-April; }
\end{array} \\
& U=2 \begin{array}{l}
\text { if the respondent's mother was born in May- } \\
\text { August; and }
\end{array} \\
& \begin{array}{ll}
\text { if the respondent's mother was born in } \\
\text { September-December. }
\end{array}
\end{aligned}
$$

In this case, it is reasonable to assume that each $p_{i}=$ $\operatorname{Pr}(U=i)$ is approximately equal to $1 / 3$. Similarly, when $m=5$, we may define

$$
\begin{aligned}
& U=1 \text { if the last digit of the respondent's ID } \\
& \begin{array}{l}
\text { card/phone number } 1 \text { or } 2 ; \\
U=2
\end{array} \begin{array}{l}
\text { if the last digit of the respondent's ID } \\
\text { card/phone number } 3 \text { or } 4 ;
\end{array} \\
& U=3 \text { if the last digit of the respondent's ID } \\
& \begin{array}{l}
\text { card/phone number } 5 \text { or } 6 ; \\
U=5
\end{array} \\
& \begin{array}{l}
\text { if the last digit of the respondent's ID } \\
\text { card the last digit of the respondent's ID } \\
\text { card/phone number } 9 \text { or } 0 .
\end{array}
\end{aligned}
$$

In Section 2, we assumed that $q=\operatorname{Pr}(W=1)$ and all $p_{i}=\operatorname{Pr}(U=i)$ for $i=1, \ldots, m$ are known. When $m=2$ and $p_{1}$ is unknown, Liu and Tian [9] further developed a variant of the parallel model [12] for sample surveys with sensitive characteristics. When $m \geq 3$ and $q$ or/and $\left\{p_{i}\right\}_{i=1}^{m}$ is unknown, it is worthwhile to investigate the corresponding multi-category parallel model.

\section{ACKNOWLEDGEMENTS}

The authors would like to thank the Editor, an AE and two referees for their comments and valuable suggestions. GL Tian's research was fully supported by a grant (HKU $779210 \mathrm{M}$ ) from the Research Grant Council of the Hong Kong Special Administrative Region.

\section{Received 20 September 2011}

\section{REFERENCES}

[1] Abul-Ela, A. A., Greenberg, B. G. and Horvitz, D.G. (1967). A multi-proportions randomized response model. Journal of the American Statistical Association 62 990-1008. MR0217982 
[2] Agresti, A. And Coull, B. A. (1998). Approximate is better than "exact" for interval estimation of binomial proportions. The American Statistician 52 119-126. MR1628435

[3] Bourke, P. D. (1974). Multi-proportions randomized response using the unrelated question technique. Technical Report No. 74 of the Errors on Surveys Research Project. Institute of Statistics, University of Stockholm (Mimeo).

[4] Bourke, P. D. And Dalenius, T. (1973). Multi-proportions randomized response using a single sample. Report No. 68 of the Errors on Surveys Research Project. Institute of Statistics, University of Stockholm (Mimeo).

[5] Brown, L. D., CAi, T. T. And DasGupta, A. (2001). Interval estimation for a binomial proportion. Statistical Science 16 101133. MR1861069

[6] Clopper, C. J. and Pearson, E. S. (1934). The Use of confidence or fiducial limits illustrated in the case of the binomial. Biometrika 26(4) 404-413.

[7] Dempster, A. P., Laird, N. M. and Rubin, D. B. (1977). Maximum likelihood from incomplete data via the EM algorithm (with discussion). Journal of the Royal Statistical Society, Series B 39(1) 1-38. MR0501537

[8] ERIKSSOn, S. A. (1973). A new model for randomized response. International Statistical Review 41 101-113.

[9] LiU, Y. AND Tian, G. L. (2012). A variant of the parallel model for sample surveys with sensitive characteristics. Technical Report of the Department of Statistics and and Actuarial Science, The University of Hong Kong.

[10] Newcombe, R. G. (1998). Two-sided confidence intervals for the single proportion: comparison of seven methods. Statistics in Medicine $\mathbf{1 7}(8)$ 857-872.

[11] Tang, M. L., Tian, G. L., Tang, N. S. and Liu, Z. Q. (2009). A new non-randomized multi-category response model for surveys with a single sensitive question: Design and analysis. Journal of the Korean Statistical Society 38 339-349. MR2750777
[12] Tian, G. L. (2012). A new non-randomized response model: The parallel model. Technical Report of the Department of Statistics and and Actuarial Science, The University of Hong Kong.

[13] Tian, G. L., Yu, J. W., Tang, M. L. and Geng, Z. (2007). A new non-randomized model for analyzing sensitive questions with binary outcomes. Statistics in Medicine 26(23) 4238-4252. MR2405351

[14] Tian, G. L, TAng, M. L., Liu, Z. Q., TAn, M. And Tang, N. S (2011). Sample size determination for the non-randomized triangular model for sensitive questions in a survey. Statistical Methods in Medicine Research 20(3) 159-173. MR2828973

[15] Warner, S. L. (1965). Randomized response: A survey technique for eliminating evasive answer bias. Journal of the American Statistical Association 60 63-69.

[16] Williamson, G. D. And Haber, M. (1994). Models for threedimensional contingency tables with completely and partially cross-classified data. Biometrics 50 194-203. MR1279436

[17] Yu, J. W., Tian, G. L. And Tang, M. L. (2008). Two new models for survey sampling with sensitive characteristic: Design and analysis. Metrika 67 251-263. MR2390876

Yin Liu

Department of Statistics and Actuarial Science

The University of Hong Kong, Pokfulam Road

Hong Kong

P. R. China

E-mail address: liuyin31@hku.hk

Guo-Liang Tian

Rm 520, Meng Wah Complex, Pokfulam Road

Hong Kong

P. R. China

E-mail address: gltian@hku.hk 